\title{
Routes to chaos in high-dimensional dynamical systems: A qualitative numerical study
}

\author{
D. J. Albers ${ }^{a}$,b J. C. Sprott ${ }^{a}$ \\ ${ }^{a}$ Department of Physics, University of Wisconsin, Madison; 1150 University \\ Avenue, Madison, WI 53706-1390 \\ ${ }^{\mathrm{b}}$ Santa Fe Institute; 1399 Hyde Park Road, Santa Fe, NM 87501
}

\begin{abstract}
This paper examines the most probable route to chaos in high-dimensional dynamical systems in a very general computational setting. The most probable route to chaos in high-dimensional, discrete-time maps is observed to be a sequence of Neimark-Sacker bifurcations into chaos. A means for determining and understanding the degree to which the Landau-Hopf route to turbulence is non-generic in the space of $C^{r}$ mappings is outlined. The results comment on previous results of Newhouse, Ruelle, Takens, Broer, Chenciner, and Iooss.
\end{abstract}

Key words:

PACS: 05.45.-a 05.45.Tp 89.75.-k 89.20.Ff

In their first edition of Fluid Mechanics [26], Landau and Lifschitz proposed a route to turbulence in fluid systems. Since then, much work, in dynamical systems, experimental fluid dynamics, and many other fields has been done concerning the routes to turbulence. In this paper, we present early results from the first large statistical study of the route to chaos in a very general class of high-dimensional, $C^{r}$, dynamical systems. Our results contain both some reassurances based on a wealth of previous results and some surprises. We conclude that, for high-dimensional discrete-time maps, the most probable route to chaos (in our general construction) from a fixed point is via at least one Neimark-Sacker bifurcation, followed by persistent zero Lyapunov exponents, and finally a bifurcation into chaos. We observe both the Ruelle-Takens

Email addresses: albers@santafe.edu (D. J. Albers), sprott@physics.wisc.edu (J. C. Sprott).

URLs: http://www.santafe.edu/ albers (D. J. Albers), http://sprott.physics.wisc.edu/sprott.htm (J. C. Sprott). 
scheme as well as persistent $n$-tori, where $n \leq 2$ before the onset of chaos. The point of this report is two-fold. First, we propose a manageable set of functions that will yield a better general understanding of high-dimensional routes to chaos in a practical sense. Second we provide a survey of the routes to chaos in this proposed set of mappings. The goal is to select a common set of mappings that exists someplace between real-world examples (the set we propose can approximate a very general set of real world examples), and the low-dimensional cases considered in rigorous mathematics.

Begin with an ordinary differential equation in $R^{k}$ with a single real parameter $\mu, \frac{d v}{d t}=F(\mu, U)$ where $F$ is as smooth as we wish and $U \subset R^{k}$ is compact. At $\mu_{0}$ there exists a fixed point, and at $\mu_{c}, \mu_{0}<\mu_{c}, F$ is chaotic. The bifurcation sequence proposed by Landau [26] and Hopf [20] is the following: as $\mu$ is increased from $\mu_{0}$ there will exist a bifurcation cascade of quasi-periodic solutions existing on higher and higher dimensional tori until the onset of "turbulence." In other words, the solutions would be of the following type, $x_{\mu_{1}}(t)=f\left(\omega_{1}, \omega_{2}\right), x_{\mu_{2}}(t)=f\left(\omega_{1}, \omega_{2}, \omega_{3}\right), \ldots, x_{\mu_{k-1}}(t)=f\left(\omega_{1}, \omega_{2}, \ldots, \omega_{k}\right)$ for $\mu_{i}<\mu_{i+1}$, and where none of the frequencies are rationally related. However, Landau and Hopf's notion of turbulence was high-dimensional, quasi-periodic flow. Ruelle and Takens [33] proposed both an alternative notion of turbulence (the strange attractor) and an alternative route to turbulence in a now famous paper. Ruelle and Takens claimed that the Landau path was highly unlikely from a topological prospective. The basis for their claim originates in the work of Peixoto [30] who has shown that quasi-periodic motion on $T^{2}$ (the 2-torus) is non-generic ${ }^{1}$ in the set of $C^{r}$ vector fields. However, Peixoto's theorem applies only to flows on $T^{2}$ and not $T^{k}$ for $k>2$. For diffeomorphisms of the circle, irrational rotations make up the full Lebesgue measure set of rotations. Suspensions of such diffeomorphisms correspond to quasi-periodic motion on $T^{2}$. It would seem that quasi-periodic motion of flows on $T^{2}$ would be high measure, however, there is not a one-to-one correspondence between flows on $T^{2}$ and discrete-time maps of the circle (e.g. the Reebs foliation or [29]). Further, quasi-periodic orbits of diffeomorphisms on the circle are structurally unstable. For flows on $T^{2}$, the structurally stable, hyperbolic periodic orbits are topologically generic; however, it is likely that quasi-periodic orbits are common in a measure theoretic sense on $T^{2}$. There remain many open questions regarding bifurcations of periodic orbits, for example, a list of codimension 2 bifurcations and their status can be found at the end of section (9.1), page 397 of [25]. How this will all play out in practice is unclear and comprises a good portion of motivation for our study. Ruelle and Takens then went on to prove two results for flows relevant to this report. The first is a normal form theorem for the "second" Hopf bifurcation for vector fields, or the "first" Hopf bifurcation for maps (often referred to as the Neimark-Sacker

\footnotetext{
1 A property is generic if it exists on subset $E \subset B$, where $E$ contains a countable intersection of open sets that are dense in the original set $B$.
} 
bifurcation [34] [27]). This theorem gives a normal form analysis of the bifurcation of an invariant circle of a flow, but it does not state the type of dynamic that will exist upon the loss of stability of the invariant circle. The second relevant result was that, given a quasi-periodic solution $f\left(\omega_{1}, \ldots, \omega_{k}\right)$ on $T^{k}, k \geq 4$, in every $C^{k-1}$ small neighborhood of $\left(\omega_{1}, \ldots, \omega_{k}\right)$, there exists an open set of vector fields with a strange attractor (prop. 9.2 [33]). These results were extended by Newhouse, Ruelle and Takens [28] who proved that a $C^{2}$ perturbation of a quasi-periodic flow on $T^{3}$ can produce strange (axiom A) attractors, thus reducing the dimension to three for tori with quasi-periodic solutions for which an open set of $C^{2}$ perturbations yield strange attractors. The basic scheme used by Takens, Ruelle and Newhouse was to first prove a normal form theorem from periodic orbits to 2-tori in vector fields and then prove something about how the 2-tori behave under perturbations - showing that bifurcations of $m$-tori to $(m+1)$-tori will yield chaos since 3 -tori can be perturbed away to axiom A chaotic attractors.

If the story were only as simple as a disagreement between topological and measure theoretic viewpoints, we would be in good shape. However, out of the complexity of the dynamics and the difficulties posed by bifurcation theory regarding what happens to bifurcations of resonant periodic orbits and quasiperiodic orbits, the field of quasi-periodic bifurcation theory was born [10] [7]. We will not attempt to discuss the history from where the above leaves off to the current state. For those interested, see [8], [12], [23], [13], [9], [24], and [22]. The question regarding the most common route to turbulence is, in any but a very select set of specific examples, still an open and poorly defined question. Analytically piecing together even what types of bifurcations exist en route to chaos has been slow and difficult. We contribute to the existing partial solution in the following ways: we will provide a framework for numerical analysis that does not have a priori tori built in; we will provide evidence that the dominant scenario with respect to the cascade of bifurcations leading to chaos in many high-dimensional dynamical systems will consist of various bifurcations between periodic orbits of high-period and quasi-periodic orbits. Moreover, flip and fold bifurcations (due to real eigenvalues) occur with less and less frequency as the dimension of the dynamical system is increased.

Let us begin by arguing why we believe that, as the dimension of the dynamical system is increased, flip, fold, any bifurcations due to real eigenvalues, and strong resonance bifurcations will be vanishingly rare and the path to chaos from a fixed point in parameter space will consist of a soup of various periodic orbits with high-period and quasi-periodic orbits. Our intuition is born from results in the random matrix theory of Girko [17], Edelman [14], and Bai [4]. The basic idea of their work is the following: given a square matrix whose elements are real random variables drawn from a distribution with a finite sixth moment, in the limit of infinite dimensions, the normalized spectrum (or eigenvalues) of the matrix will converge to a uniform distribution on 
the unit disk in the complex plane. It is worth noting that the convergence in measure is not absolutely continuous with respect to Lebesgue measure. Nevertheless, if the Jacobian of the map at the "first" bifurcation point (i.e. the bifurcation from the fixed point) is a high-dimensional matrix whose elements have a finite sixth moment, it is reasonable that the bifurcation would be of type Neimark-Sacker (via a complex eigenvalue) instead of a flip or fold, with probability approaching unity as the dimension goes to infinity. Following this line of thought, we, with W. Davis Dechert, provided strong numerical evidence that the first bifurcation in typical high-dimensional discrete-time dynamical systems would be that of the Neimark-Sacker type [3]. Extending this analytically for the $k^{\text {th }}$ bifurcation before the onset of chaos where $k>1$ is made difficult (a proof for the $k=1$ case is not difficult to construct) by the fact that the normal form and center manifold theory for quasi-periodic bifurcation theory is a long way from providing a general form(s) about which the results of the random matrix theory could be applied (the codimension 2 situation is not complete yet, and the codimension 3 case is even further from completion). However, it is assumed that, in the end, most bifurcations of periodic and quasi-periodic orbits can be captured by some sort of Taylor series expansion (via a vector field approximation or a suspension). Though the linear term of the Taylor expansion will be degenerate and the outcome of the bifurcation will be determined by contributions of higher-order terms, the degeneracies in the linear term of the Taylor polynomial will nevertheless be of complex eigenvalues - leading to some sort of a bifurcation (yet to be understood) from quasi-periodic or periodic orbits to other quasi-periodic or periodic orbits.

A full statistical study is hampered by numerical accuracy issues which may be fundamental problems associated with the existence of neutral directions. We will present the most clear picture of the prototypical route to chaos in high dimensions for our set of functions via a prototypical example. For the understanding reached in this report, we will employ bifurcation diagrams, phase plots, the largest Lyapunov exponent and the full Lyapunov spectrum. Numerical issues related to the Lyapunov spectrum are the main problem with respect to the statistical study, and we will discuss and display such problems. For information regarding why the numerical problems (such as truly infinite convergence times) we encounter may be unavoidable, see [31] and [11] for the latest with regard to the existence of zero Lyapunov exponents and [18] [19] with respect to the linear algebra issues with computing them. At the end of the report we will note the numerous extensions our current line of study suggests.

The example we will present is typical (we will discuss what is not typical about our example during the analysis) amongst the 500 or so cases (with this number of parameters and dimensions) we have observed in the sense that between the first bifurcation and the onset of chaos, the only type of orbit 
that exists is either quasi-periodic or periodic orbits with periods high enough such that they are indiscernible from quasi-periodic orbits. It is worth noting that the route to chaos we discuss in this report, which we believe is the typical route in high dimensions, is considerably different from what is observed at low dimensions. For an intuitive feel for the lower-dimensional cases, see [3].

A primary tool of analysis will be the Lyapunov exponent spectrum ([6] [32]), since it is a good measure of the tangent space of the mapping along its orbit. Negative Lyapunov exponents correspond to global stable manifolds or contracting directions; positive Lyapunov exponents correspond to global unstable manifolds or expanding directions and are ([32]), in a computational framework, the hallmark of chaos. A zero exponent corresponds to a neutral direction (although the story in this case is significantly more complicated, see [11]); when the largest Lyapunov exponent for a discrete-time map is zero and all other exponents are less than zero, then there exists a neutral direction. Lyapunov exponents relate to quasi-periodic orbits and rotations in the following way, neutral rotating directions correspond to a zero Lyapunov exponent, and high-period orbits correspond to pairs of negative Lyapunov exponents. If the largest exponent is zero (while all others are negative), then there exists a quasi-periodic orbit on the circle (on $T^{2}$ in the flow). If two exponents are zero, then there is a full 2-torus in discrete-time (a 3-torus in the flow), and so on (for specific examples of high-dimensional tori see [9]). With respect to the codimension 2 bifurcations, there can also be as many as 10 possibilities for zero Lyapunov exponents at bifurcation points (again see page 397 of [25]), some of which correspond to real and complex conjugate pairs of eigenvalues. Besides the full Lyapunov spectrum algorithm, we will also use an independent version of a calculation of the largest Lyapunov exponent a la Wolf et. al [38]. Upon analysis of the example, it will be obvious why we need such a calculation.

The discrete-time mappings we will consider are single-layer, feed-forward neural networks of the form

$$
x_{t}=\beta_{0}+\sum_{i=1}^{n} \beta_{i} \tanh \left(s \omega_{i 0}+s \sum_{j=1}^{d} \omega_{i j} x_{t-j}\right)
$$

which is a map from $R^{d}$ to $R$. In eq.(1), $n$ represents the number of hidden units or neurons, $d$ is the input or embedding dimension of the system which functions simply as the number of time lags, and $s$ is a scaling factor on the weights. The parameters are chosen in the following way: $\beta_{i}, w_{i j}, x_{j}, s \in R$, where the $\beta_{i}$ 's and $w_{i j}$ 's are elements of weight matrices (which we hold fixed for each case), $\left(x_{0}, x_{1}, \ldots, x_{d}\right)$ represent initial conditions, and $\left(x_{t}, x_{t+1}, \ldots, x_{t+d}\right)$ represent the current state of the system at time $t$. We assume that the $\beta$ 's are iid uniform over $[0,1]$ and then re-scaled to satisfy $\sum_{i=1}^{n} \beta_{i}^{2}=n$. The $w_{i j}$ 's are iid normal with zero mean and unit variance. The $s$ parameter is a real 


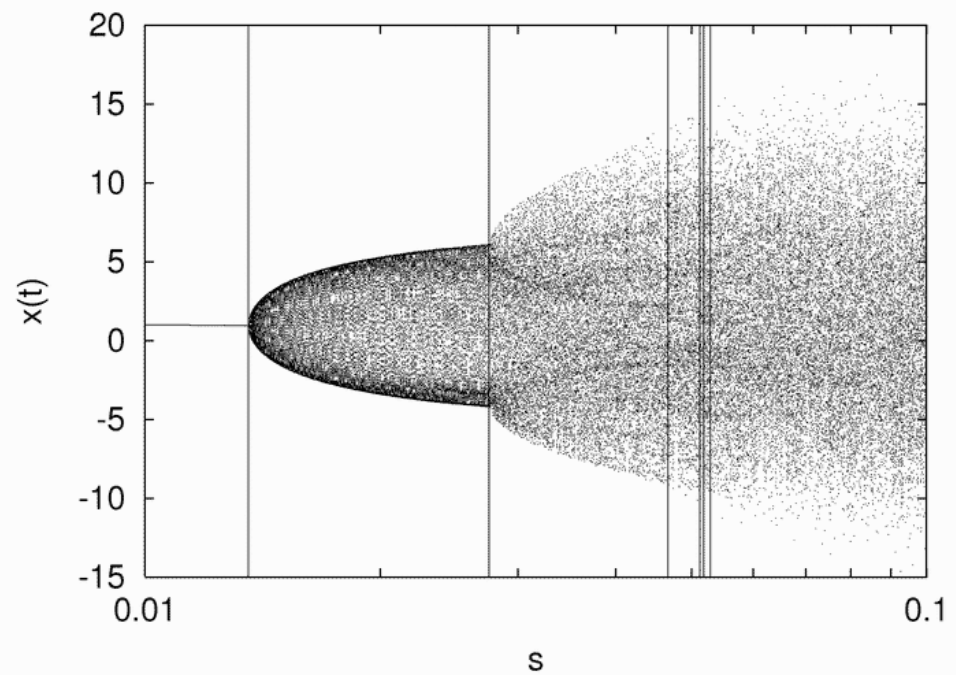

Fig. 1. Bifurcation diagram for a typical network; $n=32, d=64$.

number and can be interpreted as the standard deviation of the $w$ matrix of weights. The initial $x_{j}$ 's are chosen iid uniform on the interval $[-1,1]$.

We would like to make a few notes with respect to the squashing function, $\tanh ()$. For $x$ very near 0 , the $\tanh (x)$ function is nearly linear. Moreover, $\tanh (x)$, for $|x| \gg 1$, will tend to behave much like a binary function $( \pm 1)$. Thus the scaling parameter $s$ will provide a unique bifurcation parameter that will sweep from linear ranges to highly non-linear ranges, to nearly binary ranges - fixed points to chaos to what seem like periodic cycles. In this report however, we will cease consideration of our networks long before the neurons become saturated.

Scalar neural networks like these are "universal approximators," meaning they can approximate many very general spaces of mappings (e.g. any $C^{r}$ mapping and its derivatives to arbitrary accuracy, given enough neurons). That scalar neural networks can approximate the mappings we are interested in is a topic addressed in [21]. Combining the approximation theorems of Hornik et al [21] and the time-series embedding results of Takens [36] shows the equivalence between our neural networks and the dynamical systems from compact sets in $R^{n}$ to themselves (for specific arguments along these lines, see [1]).

To circumvent the numerical stability issues that will be apparent shortly, we are required to consider each network individually with four types of figures: the largest Lyapunov exponent independently computed of the full spectrum (it is considerably more numerically stable than the algorithm for the full spectrum); a standard bifurcation diagram; the full Lyapunov spectrum computed in the standard manner; and phase-space diagrams.

Our choice of the number of neurons and the number of dimensions is based 


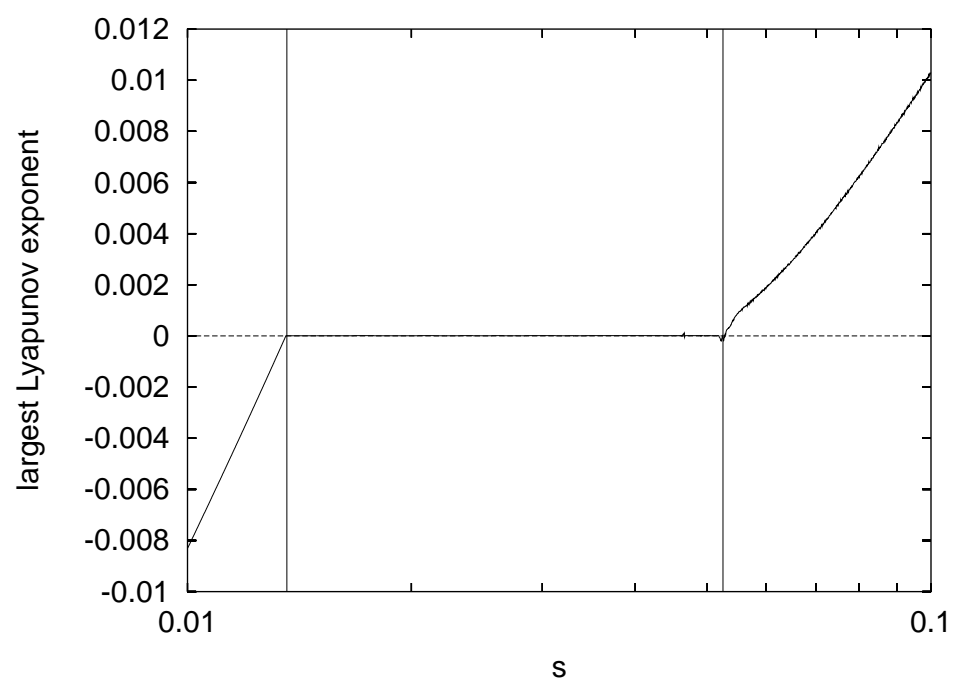

Fig. 2. The Largest Lyapunov for a typical network; $n=32, d=64$.

on Fig. (1) of [3] and the compromise required by computational time limits. Considering Fig. (1) of [3], 32 neurons puts our networks deeply in the region of the set of neural networks that correspond to highly complicated and chaotic dynamics. The dimension, 64 was chosen because it was the highest dimension for which we could reliably compute the nearly 500 cases we surveyed. The compute time increases as a power of the dimension. Thus as this time, we had too few cases of $d=128$ and $d=256$ to make conclusive statements.

Beginning with Fig. (1), the standard bifurcation diagram, there are four important features to notice. The first feature is the first bifurcation, which occurs at $s=0.0135$ from a fixed point to some type of limit cycle or torus. A secondary bifurcation is clearly visible at $s=0.02755$, the nature of this bifurcation is entirely a mystery from the perspective of Fig. (1). Chaos seems to onset near $s=0.05$, and has definitely onset by $s=0.06$, however the exact location is difficult to discern. Lastly, all of the dynamics between the fixed point and chaos are some sort of $n$-torus $(n \geq 0)$ type behavior.

Next, let us consider Fig. (2) — the largest Lyapunov exponent versus variation in $s$ for the same case as Fig. (1). Again, as expected, we see the first bifurcation at $s=0.0135$, in agreement with the bifurcation diagram. Figure (2), however, gives a clear picture of the onset of chaos, which occurs at $s=0.05284$. The largest exponent is near zero between the first bifurcation and the onset of chaos, providing evidence for the existence of at least one persistent complex conjugate pair of eigenvalues with modulus one (assuming a Jacobian can be constructed) — e.g. a persistent quasi-periodic orbit.

Considering Fig. (2), near the onset of chaos the exponent becomes negative over a very short $s$ interval. Ignoring all the intermediate bifurcations, let us briefly consider the onset of chaos via Fig. (3). Considering this figure, there 


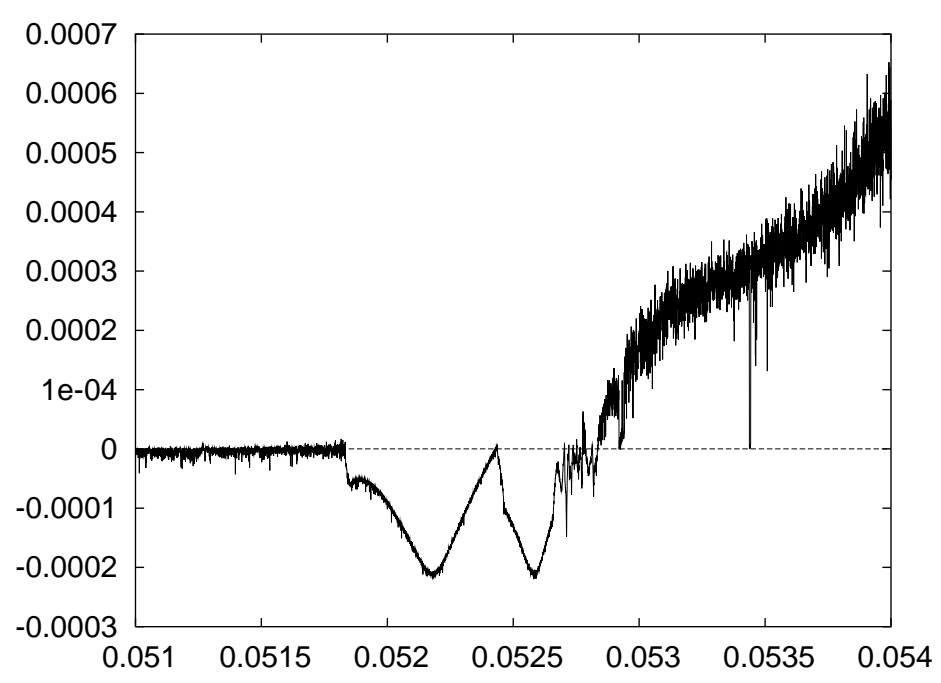

Fig. 3. The Largest Lyapunov at the onset of chaos.
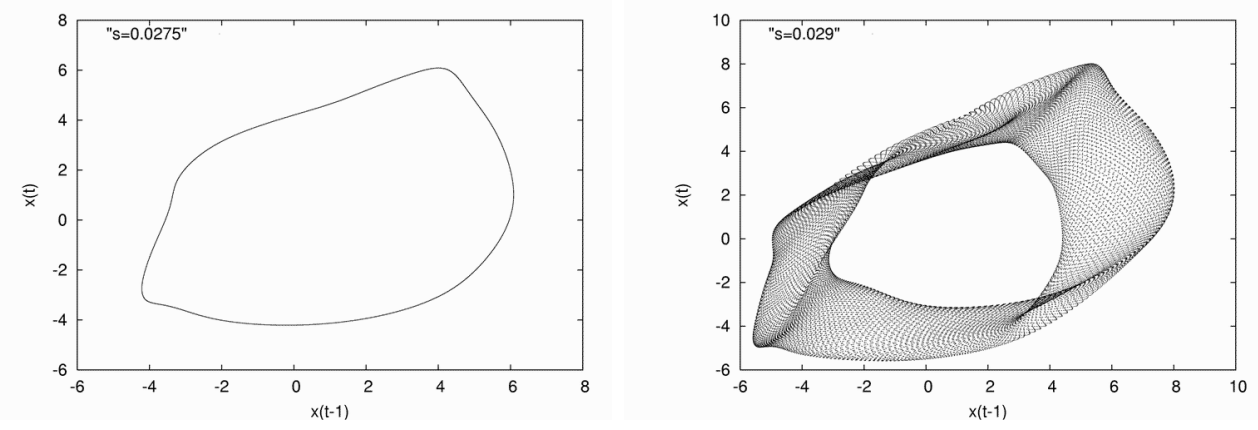

Fig. 4. Phase plots on either side if the $2^{n d}$ bifurcation, $s=0.0275$ and $s=0.029$ respectively. The bifurcation occurs at $s \sim 0.02754$.

is an apparent periodic orbit, followed but what might be a period doubling bifurcation, followed by what could be a complicated bifurcation structure. Besides noting this for general interest and completeness, we will refrain from a further discussion of this small interval since this behavior seems to disappear for high-dimensional networks and is not particularly related to the point of this report.

We will begin our presentation of phase-space figures at the second bifurcation. The second bifurcation is the obvious in the bifurcation diagram, corresponding to the rapid change in the attractor size near $s \sim 0.0275$. The locations and nature of these bifurcations can't be determined by a consideration of the Lyapunov spectrum or the largest Lyapunov exponent, although considering the Lyapunov spectrum it is clear that such a bifurcation does occur. The second bifurcation appears to be from a 1-torus to a 2-torus as shown in Fig. (4).

The phase-space plots on either side of the third bifurcation, which is not 

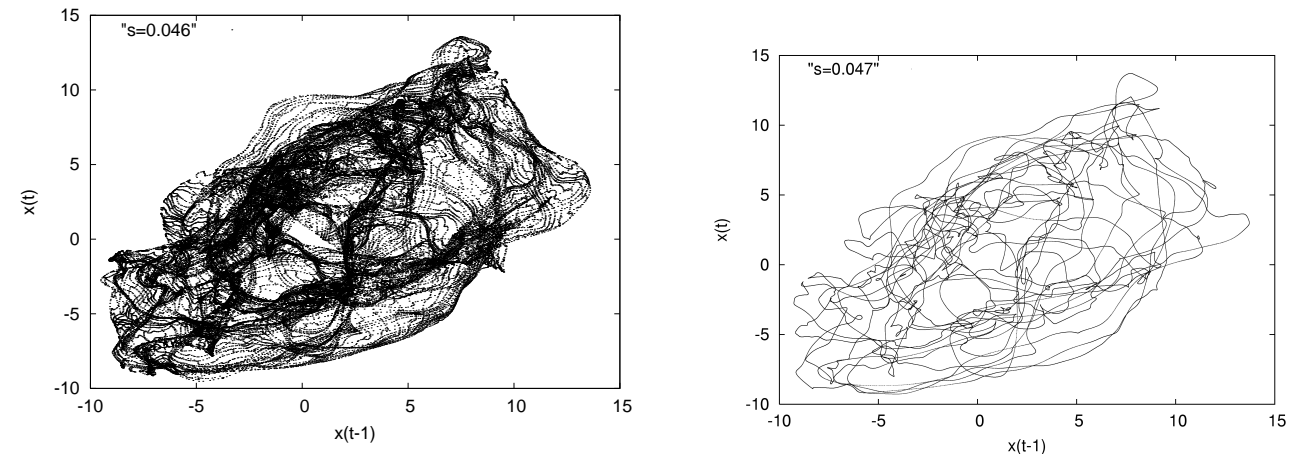

Fig. 5. Phase plots on either side if the $3^{r d}$ bifurcation, $s=0.046$ and $s=0.047$ respectively. The bifurcation occurs at $s \sim 0.04667$
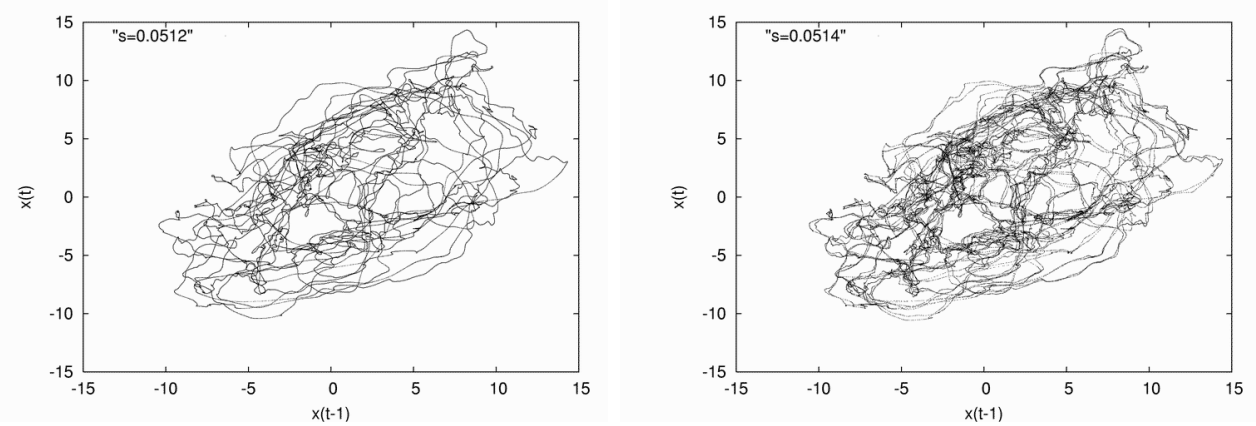

Fig. 6. Phase plots on either side of the $4^{\text {th }}$ bifurcation, $s=0.0512$ and $s=0.0514$ respectively. The bifurcation occurs at $s \sim 0.05124$

clearly apparent in the bifurcation diagram or in the Lyapunov spectrum as we will see, is depicted in Fig. (5). By this point, the smooth looking toruslike object from Fig. (4) has become "kinked" significantly before the third bifurcation. At the third bifurcation, the torus-like, two-dimensional object becomes a one-dimensional object. Thus the third bifurcation is from a 2torus to a 1 -torus and occurs at $s \sim 0.0466$. The 1 -torus is a severely "kinked" quasi-periodic orbit.

Considering the phase-space plots on either side of the fourth bifurcation in Fig. (6), one might conclude that there our example has undergone a period doubling of the quasi-periodic orbit. An analytical explanation of such a bifurcation is yet an open problem, but it is likely a "Neimark-Sacker-Flip" bifurcation. We will refrain from a further discussion of this bifurcation, directing the interested reader to chapter 9 of [25].

We will refrain from showing figures for the fifth bifurcation, simply noting that it is a bifurcation from this quasi-periodic orbit on the 1-torus to a highperiod, periodic orbit. Rather, we will skip to what we will call the sixth bifurcation. Figure (7) demonstrates the final bifurcation into chaos from a high-period, periodic orbit. However, in our particular example, considering Fig. (3), just before the onset of chaos, there is a likely a sequence of bifurca- 

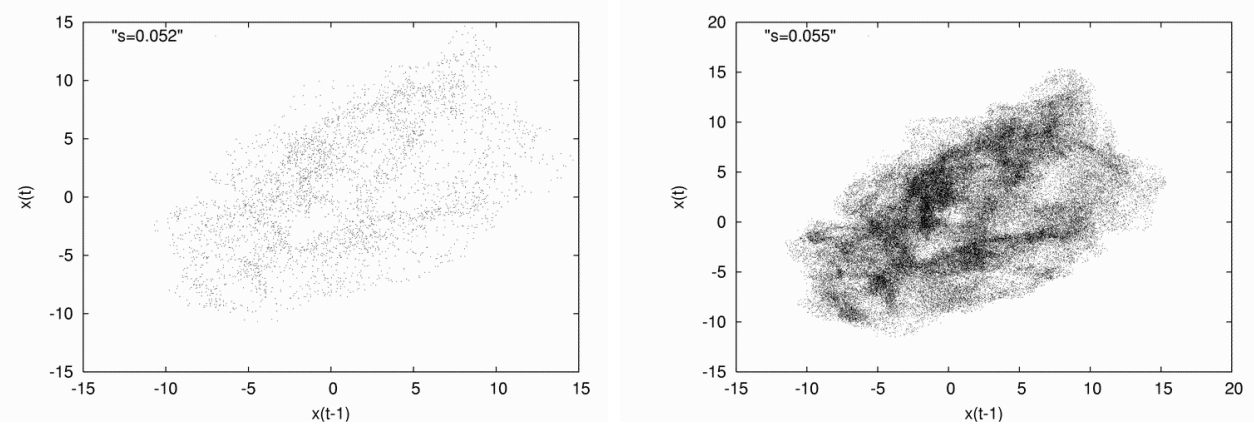

Fig. 7. Phase space plot near the $6^{\text {th }}$ bifurcation $-s=0.052$ and $s=0.053$ respectively. The bifurcation occurs at $s \sim 0.05294$.

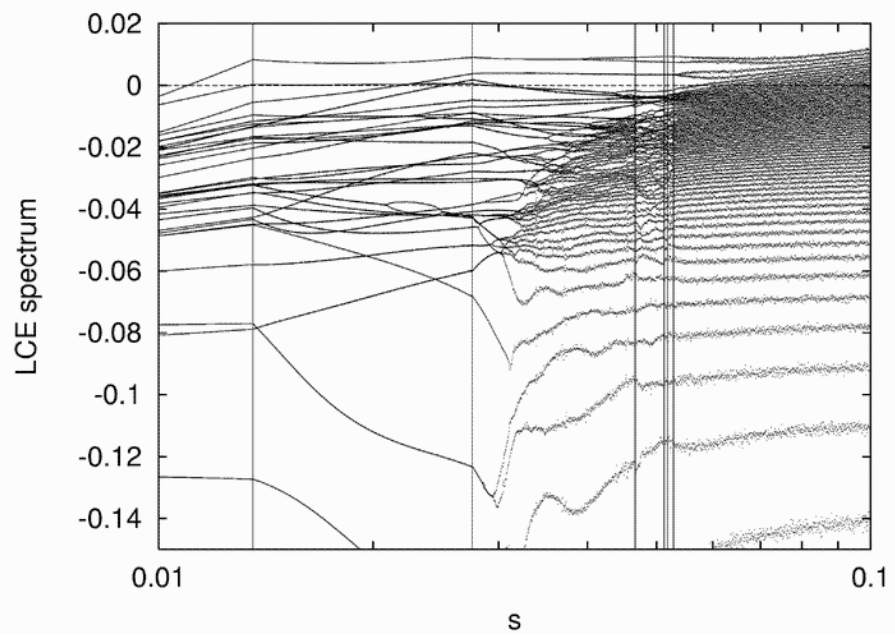

Fig. 8. Lyapunov spectrum the (typical) network; $n=32, d=64$.

tions. We will not belabor this further, as little insight is gained from a further consideration, and this sequence of bifurcations just before the onset of chaos appears to be increasingly rare as the dimension increases.

Before discussing what can be gleaned from the Lyapunov spectrum data, we must make a few comments with respect to the data plotted in Fig. (8). If one were to pick an $s$ value along the $s$ interval $(0,0.03)$ and count the number of Lyapunov exponents, it is likely that one could often only identify roughly half the number of exponents that might be expected. There is a simple reason for this, if one were to consider the eigenvalues of the variational differential equation, ${ }^{2}$ nearly all the eigenvalues are complex conjugate pairs, and hence the pairs of nearly equal Lyapunov exponents. This in a sense, implies a lot of local rotation, but as this can depend upon the coordinate system, little can be

2 See page 36 of [5] for a definition of the variational differential equation. In practical terms, we are referring to something akin to the eigenvalues of the local "Jacobian" of the network at a specific time. 
said in this regard. Nevertheless, from a practical point of view, this implies sink behavior in all directions. The point is that the "largest" exponent is actually pair of nearly equal exponents. This is true of at least the ten largest "exponents," and often this is true of all of the "exponents" on $(0,0.03)$. The Neimark-Sacker style bifurcations to periodic orbits and tori are due to those pairs of exponents. If the bifurcations were of real eigenvalues, there would not be pairs of Lyapunov exponents of equal magnitude.

On the $s$ interval $\sim(0,0.03)$, nearly all the exponents come in pairs, all the lines represent two exponents. Much of this is related to the numerical stability issues related to the linear algebra engines used to calculate the exponents. This is a fundamental and common problem in matrices with many nearly equal, and hence degenerate eigenvalues. This issue lies in the standard algorithm's ability to accurately orthogonalize the eigenvalues and eigenvectors, for more information see [18] and [19]. At $s \sim 0.03$ the negative exponents begin to split apart. This cascade continues to occur from the most negative exponents to the largest exponents, in that order, up to the onset of chaos. By the onset of chaos, there exists 64 distinct exponents, one for each dimension. In fact, by $s \sim 0.052$, all the exponent pairs are observably split. It is possible that this is the first time this phenomena has been observed. There are several interpretations for such behavior; we will sketch our interpretation. In the interval where the exponents exist in nearby pairs, the dynamics are dominated by rotation in nearly all eigen-directions, and the many nearly equal local eigenvalues cause numerical stability problems.

As the $s$ parameter is increased, the, rotating directions become less degenerate, and the lower exponents begin to separate. This phenomena has very little to do with the overall dynamics when the eigenvalues are strongly negative since these directions are so strongly contracting that they are not observed on the attractor after transients die out. As the $s$ parameter is further increased, more pairs of Lyapunov exponents separate until the neutral directions begin to separate - this is the break up of the tori and the onset of turbulence. ${ }^{3}$ We are displaying an example of the Ruelle-Takens scenario which contrasts the Landau and Hopf turbulence model. Strange attractors are not a collection of interacting quasi-periodic orbits or a rotating soup, but rather, distinct directions of expansion, contraction a la axiom A, and a little bit of rotation (neutral directions). This example, and Fig. (8) display this distinction in a very nice way. This cascade from all commensurate to all incommensurate Lyapunov exponents was unexpected.

The first bifurcation is relatively easy to identify in Fig. (8), it occurs at a relative maximum of the largest overlaying set of exponents in the spectrum

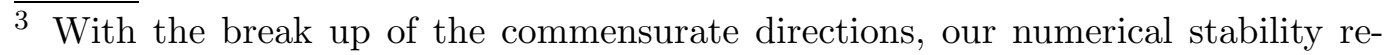
turns, see [2] or [1] for more regarding the dynamics after this transition 
at $s=0.0135$. This signals the Neimark-Sacker type first bifurcation, as expected. In our experience, the first bifurcation can be determined from the full spectrum reliably. The second bifurcation is also, relatively obvious at $s=0.02755$, and corresponds to the relative maximum of the six (three pairs) largest exponents. This would signal the existence of a torus of some type, but due to the numerical stability issues it is difficult to make a definitive statement regarding the dynamics. Nevertheless, by the second bifurcation point, there clearly exists at least a 2-torus. Near the third bifurcation the Lyapunov spectrum becomes significantly more complicated - due to the numerical accuracy issues we dare not make a claim with respect to how many exponents are actually zero; however, it appears as if there are several. The onset of chaos is vague, but chaos can clearly be identified by $s=0.8$. Likewise, considering Figs. (8) and (2), the respective largest Lyapunov exponents begin to be indistinguishable at $s=0.08$. The secondary bifurcations cannot be identified, however, and it is this problem that has hindered the full statistical study.

The previous information can be summarized in the following table for which the location of the various bifurcations is given as observed via the bifurcation diagram, the largest Lyapunov exponent, the phase space diagrams, and the Lyapunov spectrum:

\begin{tabular}{|l|l|l|l|l|l|l|}
\hline $\begin{array}{l}\text { Bif. } \\
\text { number }\end{array}$ & $\begin{array}{l}s \text { at the } \\
1^{\text {st }}\end{array}$ & $\begin{array}{l}s \text { at the } \\
2^{\text {nd }}\end{array}$ & $\begin{array}{l}s \text { at the } \\
3^{\text {rd }}\end{array}$ & $\begin{array}{l}s \text { at the } \\
4^{\text {th }}\end{array}$ & $\begin{array}{l}s \text { at the } \\
5^{\text {th }}\end{array}$ & $\begin{array}{l}s \text { at the } \\
6^{\text {th }}\end{array}$ \\
\hline $\begin{array}{l}\text { Largest } \\
\text { Lya- } \\
\text { punov } \\
\text { expo- } \\
\text { nent }\end{array}$ & 0.0135 & - & - & - & 0.05183 & 0.05289 \\
\hline $\begin{array}{l}\text { Bif. dia- } \\
\text { gram }\end{array}$ & 0.0135 & 0.02755 & - & - & - & 0.0525 \\
\hline $\begin{array}{l}\text { Phase } \\
\text { space } \\
\text { diagram }\end{array}$ & 0.0135 & 0.02754 & 0.04667 & 0.05124 & 0.05183 & 0.05289 \\
\hline $\begin{array}{l}\text { Lyapunov } \\
\text { spec- } \\
\text { trum }\end{array}$ & 0.0135 & 0.02755 & - & - & - & 0.053 \\
\hline $\begin{array}{l}\text { Transition } \\
\text { to: }\end{array}$ & $T^{1}$ & $T^{2}$ & $T^{1}$ & $T^{1}$ & $T^{0}$ & Chaos \\
\hline
\end{tabular}

Putting this all together into a unified picture, it is likely that the first bifurcation, of type Neimark-Sacker, occurs at $s=0.0135$. The second bifurcation 
occurs at $s=0.02754$ is a bifurcation from $T^{1}$ to $T^{2}$. The third bifurcation, which occurs at $s=0.04667$ is a bifurcation from $T^{2}$ back to $T^{1}$. The fourth bifurcation appears to be a period-doubling like bifurcation from $T^{1}$ to $T^{1}$. The fifth bifurcation is a bifurcation from the quasi-periodic orbit to a very highperiod orbit. Following this is a very subtle sequence of bifurcations followed by the onset of chaos at $s=0.05289$.

All the other observed cases with $d=64$ were variations on a theme. We have observed more and fewer bifurcations between the first bifurcation and chaos, but all the bifurcations are relatively similar. Period-doubling cascades and such routes are rare - they occur in less than one percent of 64-dimensional networks with our weight distribution (i.e. we did not observe a period doubling route to chaos in the $500+$ networks we considered for this report). Only five percent of the first bifurcations are due to real eigenvalues, and there never exist cascades of multiple real bifurcations. The most common route to chaos we observed is a cascade of bifurcations between $T^{1}, T^{2}$, and high-period, periodic orbits; 3 -tori are rare in our experience, we never observed one in nearly 500 cases.

One strength of our methods and results is, unlike topologically based results, our neural network framework allows not only a practical means of analyzing topological results, but it contains, in a manageable way, the supposed pathological examples. Due to the mappings that neural networks can approximate, if the spectrum of Lyapunov exponents of a $d$-dimensional network is contained in $\left[\chi_{1}, \chi_{d}\right]$, then likely there exists at least one path through parameter space such that any network can be transformed into a $T^{d}$ torus with all Lyapunov exponents being zero (we haven't proved such a result, however). If one were to stratify the networks by their spectra, the aforementioned torus would be but a point along the interval $\left[\chi_{1}, \chi_{d}\right]$, and thus, in this sense, unusual. There are other stratifications of the networks that can be made, such as the map $\phi: R^{n(d+2)} \rightarrow \Sigma(\tanh ())$, where $\Sigma(\tanh ())$ is the set of neural networks with $\tanh ()$ as the squashing function. The point is, we are presenting a practical framework that yields numerical observations regarding common routes to chaos. For example, the number of constraints required to achieve a $d$-torus might be high ${ }^{4}$, and the $T^{d}$ torus would likely exist on a surface in parameter space of much lower dimension than the ambient parameter space. However, since the neural networks can approximate $m$-tori for $m \leq d$, it is possible to study the transitions, and the likelihood of such transitions, and the persistence of high-dimensional tori in a practical way. From our experience perturbing away 2-tori and limit cycles requires drastic parameter variation, however, we never observe 3 -tori consistent with the prediction of Newhouse,

$\overline{4}$ The number of constraints for a $d$-torus might also be low, as requiring area preservation, or a local Jacobian to have an average determinant equal to \pm 1 is, in a sense, a singe constraint. 
Ruelle and Takens.

As this paper is intended as a survey of our space of neural networks along the route to chaos, let us list current and future directions of work:

i. a specific numerical analysis of each of the bifurcations in this example that can be compared to the theory; specifically the second and third bifurcations which are likely of low codimension;

ii. an analytical normal form calculation - again starting with the early bifurcations along the route;

iii. a study of Lyapunov spectrum calculation technique a la [16], [15], [37], [6], or [35] - these networks form a nice set of high-dimensional mappings to study Lyapunov spectrum calculation schemes since our mappings are high dimensional, not pathological, and are not, as high-dimensional maps go, computationally intensive to use; very often, upon the presentation of a new Lyapunov spectrum computation algorithm the test cases are very low dimensional dynamical systems for which numerical stability is rarely a problem;

iv. a brute force - by hand — statistical study of the bifurcation sequences in these networks;

v. a more numerically accurate Lyapunov spectrum calculation routine that can be used for a full statistical analysis of the routes to chaos in this set of dynamical systems;

vi. a systematic investigation of the dependence on the number of neurons;

vi. a systematic investigation of the sensitivity of the weight distribution;

vii. constraints on weight distributions which lead to dynamics similar to a particular physical phenomenon, leading to a better understanding for how special a physical phenomena is relative to a general function space.

Our results help extend the current analytical results in the sense that we have a practical way of observing transitions along an interval in parameter space. Further, we present a framework such that the parameter set for which the claimed non-generic tori and persistent quasi-periodic behavior can be more concretely understood. Based on our observations, in high dimensions, the quasi-periodic route to chaos, often with a cascade of bifurcations is the dominant route. We observe what is predicted to occur according to the Ruelle-Takens route, but the bifurcation cascade is significantly more complicated than $T^{0} \rightarrow T^{1} \rightarrow T^{2} \rightarrow$ chaos. The bifurcation cascade often involves successive bifurcations between tori with dimension $\leq 2$.

We would like to thank R. A. Bayliss and W. D. Dechert for many helpful discussions and advice. D. J. Albers would like to give special thanks to J. P. Crutchfield for guidance, many fruitful discussions, wonderful insight and feedback, and support. The computing for the project was done on the Beowulf cluster at the Santa Fe Institute and was partially supported at the 
Santa Fe Institute under the Networks, Dynamics Program funded by the Intel Corporation under the Computation, Dynamics, and Inference Program via SFI's core grant from the National Science and MacArthur Foundations. Direct support for D. J. Albers was provided by NSF grants DMR-9820816 and PHY-9910217 and DARPA Agreement F30602-00-2-0583.

\section{References}

[1] D. J. Albers and J. C. Sprott. Structural stability and hyperbolicity violation in large dynamical systems. in preparation.

[2] D. J. Albers, J. C. Sprott, and J. P. Crutchfield. A dynamics stability conjecture for high-dimensional dynamical systems. in preparation.

[3] D. J. Albers, J. C. Sprott, and W. D. Dechert. Routes to chaos in neural networks with random weights. Int. J. Bif. Chaos, 8:1463-1478, 1998.

[4] Z. D. Bai. Circular law. Ann. Probab., 25:494-529, 1997.

[5] Luis Barreira and Ya. Pesin. Lyapunov exponents and smooth ergodic theory. AMS, 2002.

[6] Giancarlo Benettin, Luigi Galgani, Antonio Giorgilli, and Jean-Marie Strelcyn. Lyapunov characteristic exponents from smooth dynamical systems and for hamiltonian systems; a method for computing all of them. part 2: Numerical application. Meccanica, 15:21-30, 1979.

[7] B. Braaksma, H. Broer, and G Huitema. Toward a quasi-periodic bifurcation theory. Mem. Amer. Math. Soc., 83(421):83-175, 1990.

[8] H. Broer. Coupled hopf-bifurcations: persistent examples of $n$-quasiperiodicity determined by families of 3-jets. In Geometric methods in dynamics (I), volume 286 of Astérisque, pages 223-229, 2003.

[9] H. W. Broer, G. B. Huitema, and M. B. Sevryuk. Quasi-periodic motions in families of dynamical systems, volume 1645 of Lecture Notes in Mathematics. Springer-Verlag, 1996.

[10] H. W. Broer, G. B. Huitema, and F. Takens. Unfoldings and bifurcations of quasi-periodic tori. Mem. Amer. Math. Soc., 83(421):1-81, 1990.

[11] K. Burns, D. Dolgopyat, and Y. Pesin. Partial hyperbolicity, Lyapunov exponents and stable ergodicity. J. Stat. Phys., 108:927-942, 2002.

[12] A. Chenciner and G. Iooss. Bifurcaions de tores invariants. Arch. Rat. Mech. Anal., 69:109-98, 1979.

[13] J.-P. Eckmann. Roads to turbulence in dissapative dynamical systems. Rev. Mod. Phys., 53:643-654, 1981. 
[14] A. Edelman. The probability that a random gaussian matrix has $k$ real eigenvalues, related distributions, and the circular law. J. Multivariate Anal., 60:203-232, 1997.

[15] K. Geist, U. Parlitz, and W. Lauterborn. Comparison of different methods for computing lyapunov exponents. Prog. Theor. Phys., 83(5):875-893, 1990.

[16] Ramazan Gencay and W. Davis Dechert. An algorithm for the $n$ Lyapunov exponents of an $n$-dimensional unknown dynamical system. Physica D, 59:142$157,1992$.

[17] V. Girko. Circular law. Theory Probab. Appl., 29:694-706, 1984.

[18] G. Golub and C. Van Lorn. Matrix computations. The Johns Hopkins University Press, $3^{\text {rd }}$ edition, 1996.

[19] N. J. Higham. Accuracy and stability of numerical algorithms. SIAM, 1996.

[20] E. Hopf. A mathematical example displaying the features of turbulence. Comm. Pure App. Math, 1:303-322, 1948.

[21] K. Hornik, M. Stinchocombe, and H. White. "Universal Approximation of an Unknown Mapping and its Derivatives Using Multilayer Feedforward Networks". Neural Networks, 3:535-549, 1990.

[22] G. Iooss and M. Adelmeyer. Topics in bifurcation theory and applications. World Scientific, 1998.

[23] G. Iooss and W. F. Langford. Conjectures on the routes to turbulence via bifurcation. New York Academy of Sciences, 1980.

[24] G. Iooss and J. E. Los. Quasi-genericity of bifurcations to high dimensional invariant tori for maps. Commum. Math. Phys., 119:543-500, 1988.

[25] Y. Kuznetzov. Bifurcation Theory. Springer-Verlag, $2^{\text {nd }}$ edition, 1998.

[26] D. Landau and L. Lifshitz. Fluid Mechanics. Pergamon Press, $1^{\text {st }}$ edition, 1959.

[27] J. Neimark. On some cases of periodic motions depending on parameters. Dokl. Acad. Nauk SSSR, 129:736-739, 1959.

[28] S. Newhouse, D. Ruelle, and F. Takens. Occurrence of strange Axiom A attractors near quasiperiodic flows on $T^{m}, m \leq 3$. Comm. Math. Phys., 64:3540, 1978.

[29] J. Palis. Vector fields generate few diffeomorphisms. Bull. Amer. Math. Soc., 80:503-505, 1974.

[30] M. Peixoto. Structural stability on two-dimensional manifolds. Topology, 1:101120, 1962.

[31] C. Pugh and M. Shub. Stable ergodicity. Bull. Amer. Math. Soc., 41:1-41, 2003. 
[32] D. Ruelle. Characteristic exponents and invariant manifolds in Hilbert space. Ann. of math., 115:243-290, 1982.

[33] D. Ruelle and F. Takens. A route to turbulence. Comm. Math. Phys., 20:167192, 1970.

[34] R. Sacker. On invariant surfaces and bifurcation of periodic solutions of ordinary differential equations. Technical Report 333, New York State University, 1964.

[35] I. Shimada and T. Nagashima. A numerical approach to ergodic problems of dissapative dynamical systems. Prog. Theor. Phys., 61:1605-1635, 1979.

[36] F. Takens. Detecting atrange attractors in turbulence. In D. Rand and L. Young, editors, Lecture Notes in Mathematics, volume 898, pages 366-381, Dynamical Systems and Turbulence, Warwick, 1981. Springer-Verlag, Berlin.

[37] H. R. von Bremen, F. E. Udwadia, and W. Proskurowski. An efficient QR based method for the computation of Lyapunov exponents. Physica D, 101:1-16, 1997.

[38] A. Wolf, J. B. Swift, H. L. Swinney, and J. A. Vastano. Detemining Lyapunov exponents from a time-series. Physica D, 16:285-317, 1984. 